\title{
Presença de excipientes com potencial para indução de reações adversas em medicamentos comercializados no Brasil
}

\author{
Antonio Vinicios Alves da Silva, Said Gonçalves da Cruz Fonseca, Paulo Sérgio Dourado Arrais, \\ Eudiana Vale Francelino*
}

Departamento de Farmácia, Curso de Farmácia, Universidade Federal do Ceará

*Correspondência:

E. V. Francelino

Departamento de Farmácia

Curso de Farmácia

Universidade Federal do Ceará

Rua Capitão Francisco Pedro, 1210

60431-327 - Fortaleza - CE, Brasil

E-mail: ceface@ufc.br
Os excipientes farmacêuticos podem ser os responsáveis por inúmeras Reações Adversas a Medicamentos (RAM). O objetivo do trabalho foi identificar a presença de possiveis excipientes indutores de RAM em medicamentos comercializados no Brasil. Foram listados os 12 produtos mais vendidos no mercado brasileiro para análise. A detecção dos excipientes ocorreu a partir da consulta à composição da fórmula farmacêutica dos produtos, realizada de Agosto a Setembro/04, no Dicionário de Especialidades Farmacêuticas, sites SAC do laboratório produtor, e ficha técnica disponivel no site da ANVISA. A identificação dos excipientes, possiveis causadores de RAM, foi realizada a partir de consulta à literatura. Foram identificadas 35 apresentações farmacêuticas, 26 classificadas como medicamentos de venda livre $(71,4 \%)$ e 15 de uso pediátrico $(42,8 \%)$. Entre os excipientes identificados $(n=100)$, nove eram possíveis causadores de RAM: metilparabeno, propilparabeno, corante amarelo tartrazina, bissulfito de sódio, benzoato de sódio, lactose, cloreto de benzalcônio, sorbitol e álcool benzílico, sendo identificados em sete apresentações de uso pediátrico $(18,9 \%)$ e doze de venda livre $(32,4 \%)$. Os resultados demonstram a necessidade de maior atenção por parte dos profissionais de saúde, dos usuários de medicamentos e da avaliação pelos sistemas de farmacovigilância, da presença de excipientes como possíveis indutores de RAM.
Unitermos

- Medicamentos/reação adversa

- Excipiente farmacêutico

- Farmacovigilância

- Conservantes farmacêuticos

\section{INTRODUÇÃO}

Os fármacos raras vezes são administrados isoladamente, eles fazem parte de uma formulação combinada com um ou mais agentes não medicinais com funções de solubilizar, suspender, espessar, diluir, emulsificar, estabi- lizar, conservar, colorir, flavorizar e possibilitar a obtenção de formas farmacêuticas estáveis, eficazes e atraentes. Com o uso seletivo desses agentes não medicinais, denominados excipientes farmacêuticos, resultam formas farmacêuticas de vários tipos (Ansel et al., 2000).

Os excipientes farmacêuticos foram apontados por 
Napke (2004) como sendo os responsáveis por inúmeras reações adversas ligadas a medicamentos, um problema importante que segundo o autor não está sendo abordado de forma adequada no momento das avaliações de casos suspeitos de Reações Adversas a Medicamentos (RAM).

A relação existente entre excipientes e reações adversas foi descrita por Buck (1996), Kumar et al. (1996, 1993) e Yang (1985), que identificaram alguns destes excipientes de risco.

Este fato e a ausência de estudos no País justificam a necessidade de se conhecer que excipientes estão presentes em formulações farmacêuticas disponíveis no mercado farmacêutico nacional.

O objetivo do presente estudo foi identificar a presença de possíveis excipientes indutores de reação adversa presentes em medicamentos comercializados no Brasil.

\section{MATERIAL E MÉTODOS}

Trata-se de um estudo descritivo, realizado no Centro de Farmacovigilância do Ceará (CEFACE), pertencente ao Grupo de Prevenção ao Uso Indevido de Medicamentos (GPUIM) do Departamento de Farmácia da Universidade Federal do Ceará. Inicialmente, realizou-se levantamento em literatura nacional e internacional para a identificação dos excipientes de maneira geral e dos possíveis causadores de efeitos indesejáveis e seus riscos à saúde da população.

No mercado farmacêutico nacional, existe um grande número de produtos registrados, portanto optou-se por centralizar a pesquisa entre os de maior venda. Para tanto, foi utilizada como referência, a lista de medicamentos com maior índice de vendas no Brasil, no período de novembro/02 a novembro/03, segundo o International Market Service (IMS, Brasil, 2003), de onde foram selecionados os 12 produtos mais vendidos. A respectiva amostra de especialidades farmacêuticas representou 5,75\% do valor monetário total em vendas (US\$273.563.312,00; valor do dólar na época $\mathrm{R} \$ 2,54$ ) e 9,37\% das unidades farmacêuticas vendidas no mercado brasileiro no período do estudo. A distribuição dos 12 produtos pode ser vista no Quadro 1, por ordem decrescente de vendas e pelo nome genérico, o qual foi associado ao código do laboratório fabricante expresso por letras do alfabeto (A, B, C, D, E, F, G, $\mathrm{H}$ e I). Essa codificação foi adotada para evitar que profissionais e consumidores fiquem sugestionados a alguma mudança de uso do medicamento referido, sem prévia consulta ao prescritor e evitar que as empresas se sintam lesadas com a exposição do nome comercial do produto. Os 12 produtos mais vendidos correspondiam a 35 apresentações farmacêuticas.
A consulta bibliográfica para a coleta de informação sobre as formas e composições farmacêuticas dos produtos selecionados foi realizada de Agosto a Setembro/04, sendo utilizadas para essa etapa as seguintes fontes de informação: Dicionário de Especialidades Farmacêuticas (2004), sites e Serviço de Atendimento ao Cliente (SAC) dos fabricantes e ficha técnica do produto disponível no site da Agência Nacional de Vigilância Sanitária (ANVISA).

Na Tabela II, podem-se observar alguns dos excipientes farmacêuticos citados por Buck (1996) e relacionados como possíveis causadores de reação adversa. Os mesmos foram selecionados para essa pesquisa a partir da identificação de suas presenças nas fórmulas farmacêuticas dos produtos pesquisados.

\section{RESULTADOS}

Os 12 produtos pesquisados apresentaram 35 apresentações farmacêuticas distribuídas nas seguintes formas farmacêuticas (Tabela I): comprimidos $(\mathrm{n}=7)$; suspensão oral $(\mathrm{n}=2)$; drágeas $(\mathrm{n}=2)$; gotas $(\mathrm{n}=7)$; supositório $(\mathrm{n}=1)$; ampola $(n=2)$; emulgel $(n=1)$; aerosol $(n=1)$; comprimido efervescente $(n=3)$; bisnaga $(n=1)$; cápsula $(n=1)$; pastilha $(n=1)$; solução nasal $(\mathrm{n}=2)$; ungüento $(\mathrm{n}=1)$; comprimido dispersível $(\mathrm{n}=1)$; comprimido mastigável $(\mathrm{n}=1)$ e solução oral ( $\mathrm{n}=1)$. Dessas apresentações, 25 são classificadas como medicamentos de venda livre $(71,43 \%)$ e 15 de uso pediátrico (42,8\%), sendo 9 (60\%) dessas últimas, de venda livre.

Quanto à fonte pesquisada, a formulação farmacêutica de nove produtos foi encontrada apenas nos sites e Serviços de Atendimento ao Cliente (SAC) dos laboratórios B e H.

Das 35 apresentações pesquisadas, em duas não foi possível identificar os excipientes pelas fontes de informação sobre o medicamento propostas na pesquisa, mas, no restante das apresentações $(\mathrm{N}=33), 100$ excipientes foram identificados. Destes, nove excipientes foram considerados como possíveis causadores de reação adversa, distribuídos em sete apresentações de uso pediátrico $(18,9 \%)$ e doze de venda livre (32,4\%) (Tabela III). Sua distribuição foi a seguinte: duas continham metilparabeno, duas propilparabeno, duas corante amarelo tartrazina, duas bissulfito de sódio, quatro benzoato de sódio, duas lactose, duas cloreto de benzalcônio, cinco sorbitol e um álcool benzílico. Em algumas situações, o produto apresentava a presença de dois ou três excipientes de risco.

Em relação à descrição das concentrações dos componentes da fórmula, nenhuma quantidade dos excipientes identificados foi descrita nas bulas, sites, ou referida pelos informantes dos SAC dos produtores, não sendo possível verificar se estão dentro dos limites normalmente usados e/ou permitidos. 
TABELA I - Lista dos medicamentos mais comercializados no Brasil, formas e apresentações farmacêuticas existentes e laboratório fabricante

\begin{tabular}{|c|c|c|}
\hline Medicamentos & Forma/Apresentação farmacêutica & Laboratório fabricante \\
\hline $\begin{array}{l}\text { Diclofenaco } \\
\text { de potássio }\end{array}$ & $\begin{array}{l}\text { lo oral; Drágeas; Gotas; Supositório; Ampola; } \\
\text { mprimido dispersível; Emulgel; Aerosol. }\end{array}$ & G \\
\hline $\begin{array}{l}\text { Dipirona }+ \text { cafeína }+ \\
\text { mucato de isometepteno }\end{array}$ & Drágeas; Gotas & $\mathrm{C}$ \\
\hline Dipirona & ; Gotas (500 mg/mL) e solução oral (50 mg/mL). & E \\
\hline Paracetamol & $\begin{array}{l}\text { omprimido ( } 500 \text { mg e } 750 \text { mg); Gotas; } \\
\text { do em suspensão; Comprimido mastigável. }\end{array}$ & $\mathrm{F}$ \\
\hline $\begin{array}{l}\text { Retinol + colecalciferol }+ \\
\text { óxido de zinco }\end{array}$ & Bisnaga & B \\
\hline Vitamina $\mathrm{C}$ & $\begin{array}{l}\text { omprimido efervescente sabor laranja; } \\
\text { mão e sabor laranjaadoçado com aspartame; } \\
\text { Gotas; Cápsulas; Pastilhas. }\end{array}$ & $\mathrm{H}$ \\
\hline $\begin{array}{l}\text { Dipirona sódica }+ \\
\text { butilbrometo de escopolamina }\end{array}$ & Comprimido; Gotas; Ampola. & $\mathrm{D}$ \\
\hline Bromazepam & Comprimido (3 mg e 6 mg) & $\mathrm{H}$ \\
\hline $\begin{array}{l}\text { Cloridrato de nafazolina }+ \\
\text { cloreto de benzalcônio }+ \text { cloreto de sódio }\end{array}$ & Solução nasal & $\mathrm{I}$ \\
\hline Cloreto de benzalcônio + cloreto de sódio & Solução nasal & I \\
\hline Dimeticona & Comprimido; gotas. & A \\
\hline Mentol + cânfora + óleo de eucalipto & Ungüento & $\mathrm{B}$ \\
\hline
\end{tabular}

TABELA II - Excipientes farmacêuticos identificados como possíveis causadores de reação adversa, segundo sua função na fórmula farmacêutica

\begin{tabular}{lc}
\hline Excipientes & Função \\
\hline (1) Metilparabeno & Conservante antifúngico \\
(2) Propilparabeno & Conservante antifúngico \\
(3) Corante FD\& C amarelo ${ }^{\circ}{ }^{5}$ (tartrazina) & Corante \\
(4) Bissulfito de sódio Obs: Sulfitos em geral & Antioxidante \\
(5) Benzoato de sódio & Conservante antifúngico \\
(6) Lactose & Edulcorante \\
(7) Cloreto de benzalcônio & Conservante antimicrobiano, tensoativo \\
(8) Sorbitol & Edulcorante, umectante \\
(9) Álcool benzílico & Conservante antimicrobiano \\
\hline
\end{tabular}

Fonte: Adaptado de Buck (1996).

\section{DISCUSSÃO}

O estudo detectou a presença de nove excipientes de risco entre as 35 apresentações farmacêuticas analisadas. Entre alguns produtos verificou-se a existência de dois ou três excipientes de risco, o que pode aumentar a chance de ocorrência de uma RA. Isso demonstra que há um risco real de exposição da população aos excipientes com potencial para indução de RAM. A preocupação é ainda maior se considerarmos que boa parte destes produtos são formulações pediátricas e de venda livre.

Este fato deve ser levado em consideração pelos prescritores, na hora de escolher o medicamento para seu paciente, e pelos responsáveis em analisar a relação de causalidade entre o medicamento e a reação adversa suspeita, nos Centros de Farmacovigilância, pois podem incorrer no erro de imputar a causa da reação ao fármaco ao invés do excipiente. 
TABELA III - Lista de produtos analisados por ordem de vendas, forma/apresentação, categoria de vendas, restrição para faixa etária e excipiente (s) de risco identificado(s)

\begin{tabular}{|c|c|c|c|c|}
\hline Produto (Laboratório) & Forma/Apresentação & Categoria deVenda & $\begin{array}{l}\text { Restrição para } \\
\text { faixa etária }\end{array}$ & $\begin{array}{l}\text { Excipiente de risco } \\
\text { identificado }\end{array}$ \\
\hline Diclofenaco de potássio $(\mathrm{G})$ & Suspensão & Prescrição médica & Pediátrico & $\begin{array}{c}\text { MetilparabenoPropilparabeno } \\
\text { sorbitol }\end{array}$ \\
\hline Diclofenaco de potássio $(\mathrm{G})$ & Injetável & Prescrição médica & Adulto & Álcool benzílico \\
\hline Dipirona 500 mg/mL (E) & Gotas & Venda livre & Adulto & Tartrazina \\
\hline Dipirona 50 mg/mL (E) & Solução oral & Venda livre & Pediátrico & $\begin{array}{l}\text { Bissulfito de sódio } \\
\text { Benzoato de sódio }\end{array}$ \\
\hline Paracetamol (F) & Gotas & Venda livre & Pediátrico & $\begin{array}{c}\text { Tartrazina } \\
\text { Benzoato de sódio }\end{array}$ \\
\hline Paracetamol $500 \mathrm{mg}(\mathrm{F})$ & Comprimido & Venda livre & Adulto & Bissulfito de sódio \\
\hline Paracetamol bebê (F) & Gotas & Venda livre & Pediátrico & Benzoato de sódio / sorbitol \\
\hline Paracetamol (F) & Suspensão & Venda livre & Pediátrico & Benzoato de sódio / sorbitol \\
\hline Vitamina $\mathrm{C}(\mathrm{H})$ & Comprimido efervescente & Venda livre & Adulto & Sorbitol \\
\hline Vitamina C (H) & Pastilhas & Venda livre & Adulto & Sorbitol \\
\hline Dipirona + butiescopolamina (D) & Comprimido & Venda livre & Adulto & Lactose \\
\hline Bromazepam $(\mathrm{H})$ & Comprimido & Prescrição médica & Adulto & Lactose \\
\hline $\begin{array}{l}\text { Cloridrato de nafazolina }+ \\
\text { cloreto de sódio (I) }\end{array}$ & Solução nasal & Venda livre & Adulto & Cloreto de benzalcônio \\
\hline Cloreto de sódio (I) & Solução nasal & Venda livre & Pediátrico & Cloreto de benzalcônio \\
\hline Dimeticona (A) & Gotas & Venda livre & Pediátrico & $\begin{array}{l}\text { Metilparabeno } \\
\text { Propilparabeno }\end{array}$ \\
\hline
\end{tabular}

Em pesquisa realizada na literatura científica podese evidenciar o risco relacionado a cada um dos excipientes identificados nos produtos vendidos no mercado farmacêutico nacional. Os principais problemas relacionados a esses excipientes são descritos a seguir.

\section{Conservantes - Metilparabeno e Propilparabeno}

No estudo, foram detectadas duas formulações pediátricas (dimeticona gotas e diclofenaco de potássio suspensão), contendo os conservantes metilparabeno e propilparabeno. Segundo Jackson (1992), citado por Soni et al. (2001), a combinação de metilparabeno e propilparabeno nas formulações farmacêuticas é a forma mais utilizada, o que foi identificado pelo estudo em algumas situações.

De acordo com Soni et al. (2001), numerosos casos de reações adversas para parabenos e benzoatos, principalmente reações de hipersensibilidade, foram reportados na literatura médica, devido ao fato de suas estruturas químicas serem muito parecidas. Os parabenos após seu metabolismo são convertidos em parte ao ácido $\rho$-hidroxibenzóico, estruturalmente relacionado ao ácido acetilsalicílico, substância bem conhecida em causar reações de hipersensibilidade. Muitas dessas reações são leves e geralmente envolvem casos de reações de contato associadas com o uso de parabenos em formulações cosméticas.

\section{Conservante - Benzoato de sódio}

As formulações pediátricas paracetamol gotas, dipirona $50 \mathrm{mg}$ (solução oral), paracetamol bebê (gotas) e paracetamol suspensão apresentaram em suas formulações o conservante antifúngico benzoato de sódio, que aparece relacionado com o surgimento de erupções cutâneas como urticária imune associada à angioedema, eczema atópico, dermatites de contato e casos de reações anafiláticas (Vilaplana, Romaguera, 2003).

\section{Conservante - Cloreto de benzalcônio}

No estudo, identificou-se a presença do conservante antimicrobiano, cloreto de benzalcônio, em solução nasal de cloridrato de nafazolina associada a cloreto de sódio (uso adulto) e cloreto de sódio (uso pediátrico). Esse foi apontado por Buck (1996), como responsável por diminuição significativa da função pulmonar e reações de hipersensibilidade em pacientes asmáticos. Segundo estu- 
do realizado por Scadding (2000) observou-se que, em um número de 1.000 pacientes, 10 experimentaram reações de hipersensibilidade ao cloreto de benzalcônio, correspondendo a uma prevalência de $1 \%$.

Os achados citados por Graf (2002) demonstram também que o uso do cloreto de benzalcônio pode agravar o quadro de rinite medicamentosa induzida por descongestionantes nasais, que segundo Scadding (2000) é considerado além da rinite alérgica, um efeito adverso causado por este excipiente.

\section{Antioxidantes - Sulfitos em geral}

A presença de sulfitos foi percebida nos medicamentos analisados à base de dipirona (solução oral) e paracetamol (comprimido).

No caso do sulfito, o sintoma mais freqüente de uma reação adversa é a dificuldade para respirar. Outros sinais/ sintomas são: diarréia, náusea e vômitos, cólicas abdominais, tontura, respiração ruidosa, urticária, coceira, edema local, exantema, dificuldade para engolir, cefaléia, desmaios, mudanças da temperatura corporal, dor torácica, alterações na freqüência cardíaca, inconsciência e coma (Ansel et al., 2000). Os sintomas, normalmente, ocorrem minutos após a ingestão de alimentos ou medicações que contêm sulfito. Muitas pessoas sensíveis ao sulfito têm asma ou outras condições alérgicas.

Buck (1996) também relata para os sulfitos, uma diminuição significativa da função pulmonar e reações de hipersensibilidade em pacientes asmáticos.

\section{Edulcorante - Lactose}

A lactose foi outro excipiente encontrado em formulações analisadas pelo estudo e disponíveis no mercado nacional como a formulação à base de bromazepam e outra à base de dipirona associada à butilescopolamina. A substância é comumente utilizada como diluente nas formas farmacêuticas sólidas. A sensibilidade de pacientes sensíveis à ingestão de lactose pode variar em termos de gravidade, chegando o paciente a apresentar diarréia, flatulência, dores abdominais resultando em restrição do seu consumo após ingestão de $3 \mathrm{~g}$ ou menos (American Academy of Pediatrics Committee on Drugs, 1997).

\section{Corante - Amarelo tartrazina}

O corante tartrazina foi encontrado em dois produtos: dipirona $500 \mathrm{mg}$ (gotas) e paracetamol (gotas). No estudo, foi identificada que sua presença está ocorrendo, principalmente, em produtos destinados ao uso pediátrico.
De acordo com Buck (1996), esse excipiente é responsável por reações de hipersensibilidade em pacientes com predisposição alérgica como anafilaxia, broncoconstrição, angioedema, urticária, dores abdominais e vômitos, bem como dermatite de contato.

Numerosos corantes também são usados em produtos farmacêuticos. Vários foram associados com reações adversas graves. Um deles é o corante amarelo tartrazina, conhecido como sendo de alto risco em indivíduos intolerantes ao ácido acetilsalicílico (American Academy of Pediatrics Committee on Drugs, 1997). Aproximadamente 2 a 20\% de asmáticos são sensíveis ao ácido acetilsalicílico. Em estudos realizados por Stevenson et al. (1986) e por Simon (1984), a incidência de reação cruzada para tartrazina tem sido inferior a $2,4 \%$. Podem ocorrer reações decorrentes do uso desse corante (broncoespasmo agudo, urticária não-imunológica, eosinofilia e angioedema) em indivíduos com ou sem história de intolerância ao ácido acetilsalicílico. Raramente ocorrem reações anafilactóides não-imunológicas (American Academy of Pediatrics Committee on Drugs, 1997).

Considerando este risco, no Brasil, segundo a Resolução da Diretoria Colegiada (RDC) 137/03 da ANVISA, o medicamento que utilizar o corante amarelo tartrazina em suas formulações, deve trazer a seguinte advertência na bula e nos rótulos das embalagens secundárias: "Este produto contém o corante amarelo de tartrazina, que pode causar reações de natureza alérgica, entre as quais asma brônquica, especialmente em pessoas alérgicas ao ácido acetilsalicílico".

\section{Edulcorante - Sorbitol}

A presença de edulcorantes como o sorbitol nas formulações medicamentosas como o diclofenaco de potássico (suspensão), vitamina C (efervescente e pastilha) e paracetamol (gotas e suspensão), tem sido associada a relatos de transtornos gastrintestinais como diarréia e dores abdominais, pois é a grande quantidade de sorbitol contida nessas formulações a responsável por esse fato (Buck, 1996).

O sorbitol, assim como outros adoçantes (sacarina, aspartame e frutose), são largamente utilizados em formulações farmacêuticas, principalmente às destinadas à pediatria.

\section{Conservante - Álcool benzílico}

O diclofenaco de potássio, na forma injetável (laboratório $\mathrm{G}$ ), foi o único medicamento de uso adulto, que continha o excipiente álcool benzílico em sua formulação.

O álcool benzílico é comumente usado como preservativo em muitas soluções e medicamentos injetáveis. Segun- 
do a American Academy of Pediatrics Committee on Drugs (1997), um número de mortes neonatais e complicações metabólicas e respiratórias graves em prematuros de baixo peso ao nascer foi associado com o uso destes agentes em soluções salinas bacteriostáticas de uso endovenoso e soluções para lavagem do tubo traqueal. A incidência de mortalidade de infantes prematuros, quadro de Kernicterus e hemorragia intraventricular reduziram notadamente após descontinuação de soluções conservantes como o álcool benzílico. Em neonatos sobreviventes, expostos ao álcool benzílico, foi também encontrada associação com morbidade, incluindo paralisia e retardo no desenvolvimento.

Casos envolvendo o uso de álcool benzílico, presente em formulações injetáveis como conservante antimicrobiano, foi responsável por colapso cardiovascular neonatal caracterizado por acidose metabólica e anormalidades hematológicas, e em pacientes idosos, por relatos de reações de hipersensibilidade como dermatite de contato, náuseas e angioedema (Buck, 1996). O álcool benzílico é usado como conservante em preparações parenterais destinados ao uso em múltiplas doses, utilizando-se como veículo a água bacteriostática para injetáveis (Ansel et al., 2000).

\section{Outras considerações importantes}

O envolvimento dos excipientes como fator de risco para reações adversas a medicamentos está associado às ações como: prevenção, modificação das formulações farmacêuticas e vigilância contínua do seu uso na população em geral. A identificação de excipientes problemáticos pode levar a Indústria Farmacêutica a reavaliar seus produtos, propondo modificação das formulações farmacêuticas, por meio de solicitações junto ao órgão regulador, no caso do Brasil, a ANVISA, fato este regido pela Resolução da Diretoria Colegiada (RDC) 140/03.

Com referência à Resolução da Diretoria Colegiada (RDC) 210/04, que expressa a necessidade da menção das concentrações de todos os componentes da fórmula de um produto farmacêutico para a entrada de seu registro, não foi encontrada no estudo nenhuma concentração descrita dos excipientes presentes nas apresentações analisadas, mas, somente "quantidade suficiente para" (q.s.p), pois o fabricante não é obrigado a expressar as concentrações nos rótulos, bulas e cartuchos, provavelmente como forma de garantir o segredo industrial de seus produtos (Balbani, Stelzer, Montovani, 2006).

No caso dos dois produtos em que não foi possível identificar os excipientes em sua composição, a omissão desta informação pode gerar um fator de risco adicional para pacientes portadores de hipersensibilidade. Esta não é uma situação isolada, pois estudos realizados por
Auricchio, Batistic-Longatto e Nicoletti (2007), Balbani, Stelzer e Montovani (2006) e Barros (2000), demonstraram que as informações veiculadas em rótulos, bulas e no DEF apresentaram sérias deficiências. Isso explica a necessidade de utilização dos sites da ANVISA e dos laboratórios produtores dos medicamentos para obtenção, entre outras, de informações sobre a composição dos produtos. É consenso, entre os autores, que a falta de informações pode prejudicar a prescrição racional e o uso correto pelo paciente.

Com relação à presença de excipientes de risco em medicamentos de venda livre, verificou-se que os mesmos estão associados, principalmente, a produtos utilizados na automedicação (Pereira et al., 2007; Servidoni et al., 2007; Arrais et al., 1997), como dipirona, dimeticona, vitamina C, paracetamol, cloridrato de nafazolina associado a cloreto de benzalcônio, cloreto de sódio associado a cloreto de benzalcônio e as associações cafeína + dipirona + cloridrato de isometepteno; dipirona associada + butilbrometo de escopolamina e cânfora, mentol e óleo de eucalipto. Isto é preocupante, já que essa prática, bastante difundida no Brasil, é considerada por Honig (1995) um fator de risco para o aparecimento de reação adversa que, somado à presença desses excipientes, pode aumentar ainda mais a possibilidade de sua ocorrência.

\section{$\mathrm{O}$ risco desses produtos em pacientes pediátricos}

Outro fator condicionante de risco é o fato de muitos destes produtos serem de uso pediátrico. A incidência de efeitos adversos por medicamentos na idade pediátrica pode variar entre $0,6 \%$ a $18,0 \%$ (Santos, 2004). Por outro lado, as RAM têm sido responsabilizadas por $0,6 \%$ a $4,3 \%$ das internações hospitalares, colocando em grave risco a vida das mesmas (38\%) ou mesmo levando ao óbito. O risco pediátrico evidenciado gera cuidados adicionais como maior vigilância e orientação no uso do medicamento por parte de seus cuidadores e uma prescrição racional por parte dos prescritores.

\section{Os excipientes e o ambiente hospitalar}

As reações adversas aos excipientes que compõem o medicamento também constituem um grave problema de saúde, uma vez que 10 a $20 \%$ das internações hospitalares são decorrentes de reações adversas sem a possibilidade de excluí-los da relação de causalidade. De acordo com Wong e Sandron (2001), as RAM podem ocorrer com mais freqüência na América Latina do que em outras regiões devido ao uso elevado de medicamentos, a existência de automedicação e polifarmácia, a aquisição de medicamentos sem receita médica e a freqüente utilização de medica- 
mentos derivados de plantas medicinais. Ainda, nessa região, as notificações de RAM são escassas. Segundo os autores, isto se deve a informação equivocada por parte dos médicos sobre o tema, os quais consideram que informar sobre RAM consome seu tempo e os compromete, assim como a crença de que os medicamentos que estão no mercado são seguros e de que existe uma estreita ligação entre a ocorrência de reação adversa com o princípio ativo mais do que com o excipiente.

Neste processo de informar e orientar o usuário de medicamentos sobre sua eficácia e segurança, o profissional farmacêutico tem papel importante. A Resolução do Conselho Federal de Farmácia n ${ }^{\circ} 357 / 01$, nos termos dos Art. 31, 32 e 33, conclama o profissional para assumir seu papel como orientador quanto ao uso racional, interações medicamentosas, compreensão do próprio paciente acerca do tratamento e sua adesão, bem como o registro de todas as suas ações durante a consulta farmacêutica.

\section{CONCLUSÃO}

Os excipientes com risco de causar reações adversas foram detectados entre as formulações farmacêuticas mais consumidas no Brasil, principalmente as de uso pediátrico e de venda livre. Considerá-las como "substâncias inertes" é um grave erro. É, portanto, necessário, tornar real a sensibilização dos profissionais de saúde sobre o assunto, para que possam realizar a identificação prévia de pacientes predispostos a hipersensiblidade e alertar a população consumidora sobre os riscos, por meio da melhoria das bulas ou embalagens de medicamentos e das informações constantes em guias terapêuticos e fontes de informação de natureza comercial, como o DEF.

\section{ABSTRACT}

\section{The presence of pharmaceutical excipients as possible cause of adverse drug reactions (ADR) - Situation in Brazil}

Pharmaceutical excipients can be responsible for many ADR. The objectives of this study were to identify the presence of possible excipients as cause of ADR in drugs commercialized in Brazil. Twelve medicines with high indices of sales in Brazil, were selected to analysis. The bibliographic research about the Pharmaceutical Preparations (PP) was carried from August to September/04. The sources of information used were Pharmaceutical Specialties Dictionary, web sites and customer services from the manufacturers and technical files of the
National Agency of Sanitary Vigilance. The excipients were detected as a whole and also the compounds that may cause adverse reactions and its risks to the health. They were identified 35 PP. Of these, 26 were classified as Over-TheCounter medicines (OTC) (71.4\%) and 15 of paediatric use $(42.8 \%)$. Between the excipients identified ( $n=100), 9$ were possible causes of ADR: metylparaben, propylparaben, yellow dye tartrazine, sodium bissulfit, sodium benzoate, lactose, benzalkonium chloride, sorbitol and benzilic alcohol, being identified in 7 use in paediatric (19.0\%) and 12 of OTC $(32.4 \%)$. The results shows the need of more attention from the health professionals, the patient and the development of evaluation by the pharmacovigilance systems, of the presence of excipients as possible inductors of ADR.

UNITERMS: Drugs/ adverse reactions. Pharmaceutical excipients. Pharmacovigilance. Pharmaceutical preservatives.

\section{REFERÊNCIAS BIBLIOGRÁFICAS}

ANSEL, H. C.; POPOVICH, N. G.; ALLEN JUNIOR, L. V. Farmacotécnica: formas farmacêuticas e sistema de liberação de fármacos. 6. ed. São Paulo: Premier, 2000.568p.

AMERICAN ACADEMY OF PEDIATRICS COMMITTEE ON DRUGS (AAP). "Inactive" ingredients in pharmaceutical products: update (subject review). Pediatrics, v.99, n.2, p.268-278, 1997.

AMERICAN SOCIETY OF HOSPITAL PHARMACISTS (ASHP). 1994. Disponível em: <http://www.ashp.org/ bestpractices/medtherapy/Specific_Gdl_Peds.pdf $>$. Acesso em: 19 Jun. 2006.

ARRAIS, P.S.D.; COELHO, H.L.L.; BATISTA, M.C.D.S.; CARVALHO, M.L.; RIGHI, R.E.; ARNAU, J.M. Perfil da automedicação no Brasil. Rev. Saúde Pública, v.31, n.1, p.71-77, 1997.

AURICCHIO, M. T.; BATISTIC-LONGATTO, M. A.; NICOLETTI, M.A. Análise comparativa de embalagens secundárias e bulas de medicamentos contendo Panax ginseng C. A. Meyer. Cad. Saúde Pública, v.23, n.10, p.2295-2304, out. 2007.

BALBANI, A. P. S.; STELZER, L. B.; MONTOVANI, J. C. Excipientes de medicamentos e as informações da bula. Rev. Bras. Otorrinolaringol., v.72, n.3, p.400-406, 2006. 
BARROS, J. A. C. A(des)informação sobre medicamentos: o duplo padrão de conduta das empresas farmacêuticas. Cad. Saúde Pública, v.16, n.2, p.421-427, 2000.

BRASIL. Agência Nacional de Vigilância Sanitária. RDC n ${ }^{\circ}$ 137, de 29 de maio de 2003. Autoriza, apenas, o registro/ renovação de regulamento de medicamentos pertencentes às classes/princípios ativos, só se as bulas e embalagens contiverem a advertência pertinente. Disponível em: $<\mathrm{http}: / /$ www.anvisa.gov.br>. Acesso em: 27 out. 2004.

BRASIL. Agência Nacional de Vigilância Sanitária. RDC n ${ }^{\circ}$ 140, de 29 de maio de 2003. Estabelece regras das bulas de medicamentos para pacientes e para profissionais de saúde. Disponível em: <http://www.anvisa.gov.br $>$ Acesso em: 27 out. 2004.

BRASIL Agência Nacional de Vigilância Sanitária. RDC n ${ }^{\circ}$ 210, de 2 de setembro de 2004. Dispõe sobre a adequação dos medicamentos já registrados. Disponível em: $<$ http:/ /www.anvisa.gov.br>. Acesso em: 27 jun. 2005.

BRASIL. Agência Nacional de Vigilância Sanitária. RE n ${ }^{\circ}$ 528, de 17 de abril de 2001. Proíbe o uso de compostos mercuriais nos medicamentos e dá outras providências. Disponível em: $<$ http://www.anvisa.gov.br $>$. Acesso em: 28 jun. 2005.

BRASIL. Agência Nacional de Vigilância Sanitária. RE n ${ }^{\circ}$ 552, de 20 de abril de 2001. Determina a proibição da presença do acido bórico e o borax na composição de produtos anti-sépticos tópicos, na forma de pomadas, talcos e cremes, indicados para uso infantil, fabricados a partir desta data. Disponível em: <http:// www.anvisa.gov.br>. Acesso em: 28 jun. 2005.

BUCK, M. A guide to pharmaceutical excipients (Inert Ingredients). Pediatr. Pharmacother., v.2, n.9, p.1-5, 1996.

CONROY, S.; CHOONARA, I.; IMPICCIATORE, P.; MOHN, A.; ARNELL, H.; RANE, A.; KNOEPPEL, C.; SEYBERTH, H.; PANDOLFINI, C.; RAFFAELLI, M. P.; ROCCHI, F.; BONATI, M.; JONG, G.; DE HOOG, M.; VAN DENANKER, J. Survey of unlicensed and off label drug use in paediatric wards in European countries. $B M J$, v.320, n.7227, p.79-82, 2000.

GRAF, P. Benzalkonium chloride as a preservative in nasal solutions: reexamining the data. Respir. Med., v.95, n.9, p. $728-733,2002$.
HONIG, P.K.; GILLESPIE, B.K. Drug interation between prescribed and over-the-counter medication. Drug Saf., v.13, n.5, p. 296-303, Nov. 1995.

JACKSON, E.M. Moisturizers of today. J. Toxicol. Cutan. Ocul. Toxicol., v.11, n.3, p.173-184, 1992.

KUMAR, A.; RAWLINGS, R. D.; BEAMAN, D. C. The mystery ingredients: sweeteners, flavorings, dyes, and preservatives in analgesic/antipyretic, antihistamine/ decongestant, cough and cold, antidiarrheal, and liquid theophylline preparations. Pediatrics, v.91, n. 5, p.927, 1993.

KUMAR, A.;AITAS, A.T; HUNTERA.G;BEAMAN, D. C. Sweeteners, dyes, and other excipients in vitamin and mineral preparations. Clin. Pediatr., v. 35, n. 9, p. 443450, 1996.

LOYOLA FILHO, A. I.; UCHOA, E.; GUERRA, H. L.; FIRMO, J. O.A.; LIMA COSTA, M. F. Prevalência e fatores associados à automedicação: resultados do projeto Bambuí. Rev. Saúde Pública, v. 36, n. 1, p. 55-62, 2002.

NAPKE, E. A commitment to pharmacovigilance: 40 years on Ed Napke reflects on his life in pharmacogilance. Uppsala Rep., April, supl. 25, p.1-15, 2004.

OLIVEIRA, G.G. As reações adversas a medicamentos (RAM): o risco terapêutico. Fol. Méd., v.109, n. 1, p. 47$50,1994$.

PEREIRA, F. S. V. T.; BUCARETCHI, F.; STEPHAN, C.; CORDEIRO, R. Automedicação em crianças e adolescentes. J. Pediatr., Rio de Janeiro, v. 83, n. 5, p. 453458, 2007.

SANTOS, D. B. dos; COELHO, H. L. L. Reações adversas a medicamentos em pediatria: uma revisão sistemática de estudos prospectivos. Rev. Bras. Saude Mater. Infant., v. 4 , n. 4, p.341-349, 2004.

SCADDING, G.K. Adverse effects of benzalkonium chloride on the nasal mucosa: allergic rhinitis and rhinitis medicamentosa. Clin. Ther., v. 22, n. 7,p. 893-895, 2000.

SERVIDONI, A. B., COELHO, L., NAVARRO, M.L. et al Perfil da automedicação nos pacientes otorrinolaringológicos. Rev. Bras. Otorrinolaringol., v. 72, n. 1, p. 83-88, jan./fev. 2006. 
SONI, M.G.; BURDOCK, G.A.; TAYLOR, S.L.; GREENBERG, N.A. Safety assesment of propyl paraben: a review of the published literature. Food Chem. Toxicol., v.39, n.6, p.513-532, 2001.

TEJEDOR, N.; ZAFRA, E.; SANCHEZ, Y. Transtornos comunes de salud: autocuidado y automedicación. Aten. Primaria, v.16, n.16, p.13-18, 1998.
VILAPLANA, J.; ROMAGUERA, C. Fixed drug eruption from sodium benzoate. Contact Dermatitis, v.49, n.6, p.290-291, 2003.

WONG, A.; SANDRON, C. A. Increasing drug safety and ADR reporting in Latin America: the crucial role of poison information centers (Abstract). Toxicology, v.164, p.8, 2001.

Recebido para publicação em 07 de fevereiro de 2007 Aceito para publicação em 12 de junho de 2008 